\title{
The Effect of Ghrelin Agonist, Exercise, and Nicotine on Catalepsy in an Animal Model of Parkinson's Disease
}

\author{
Ramesh Ahmadi*, Leila Sohrabian
}

Department of Physiology, Qom Branch, Islamic Azad University, Qom, Iran

\section{A BSTRACT}

Introduction: Parkinson's disease (PD) is one of the most common neurodegenerative disorders. The progressive loss of dopaminergic neurons in the substantia nigra pars compacta (SNPC) is the principal cause of symptoms of PD. It has been suggested that ghrelin, a peptide transgenic arc, may play an important role in protecting neurons in SNPC. Furthermore, exercise improves the brain function and upregulates the expression of neurotrophic factor in the striatum. Nicotine affects different types of nicotinic receptors within the nigrostriataldopaminergic pathway. The aim of the present study was to evaluate the effect of ghrelin, exercise, and nicotine on catalepsy in reserpine-induced PD model in rats. Materials and Methods: 114 Wistar rats were divided into 19 groups. To induce PD model, reserpine (1mg/ $\mathrm{Kg}$; dissolved in glacial acetic acid solvent $0.03 \mathrm{ml}$ ) was injected intrapritoneally (i.p.) and catalepsy was assessed by test bars. Rats in exercise group were forced to have treadmill exercise ( 5 days a week for 20 minutes at a speed of 24 meters per minute at zero slope). Ghrelin was injected (i.p., $0.1 \mathrm{mg} / \mathrm{Kg}$ ) for 21 days and nicotine (i.p., $0.002 \mathrm{mg} / \mathrm{kg}$ ) was injected for 21 days. Results: The catalepsy was significantly reduced in PD rats treated with ghrelin, exercise, and nicotine. Conclusion: A combined treatment with ghrelin, exercise, and nicotine could be a potential treatment for catalepsy in patient with PD.

Key words:

1. Ghrelin

2. Exercise

3. Nicotine

4. Catalepsy

5. Parkinson Disease

*Corresponding Author: Ramesh Ahmadi

E-mail: ramahmd@yahoo.com 
اثر آتونيست كرلين، ورزش و نيكوتين بر كاتاليسى در مدل حيوانى بيمارى هاركينسون

رامش احمدى"، ليلا سهرابيان

كروه فيزيولوزى، واحد قم، دانشكاه آزاد اسلامى، قم، ايران

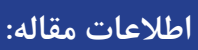

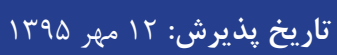

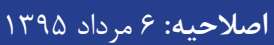

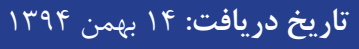

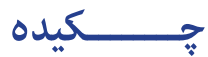

مقدمه: بيمارى يار كينسون يكى از رايجترين اختلالات تحليل برنده عصبى است. از دست رفتن تورئ تدريجى

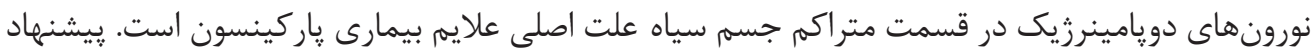

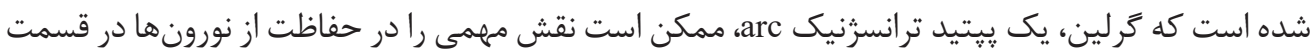

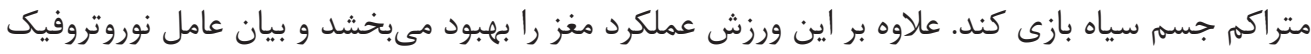

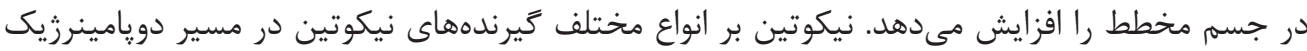

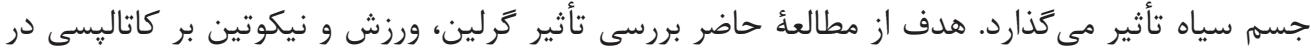

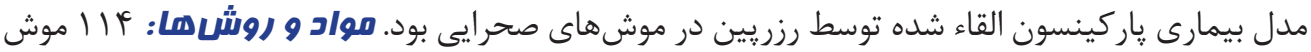

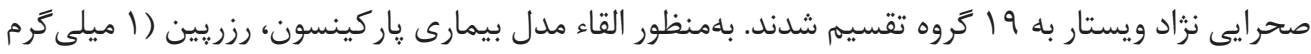

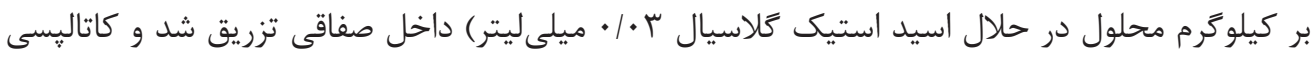

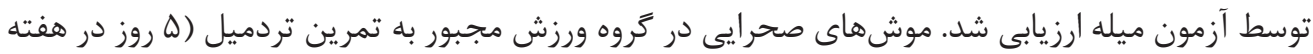

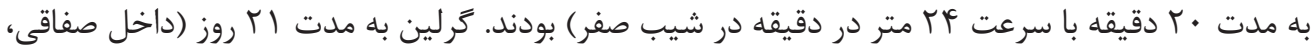

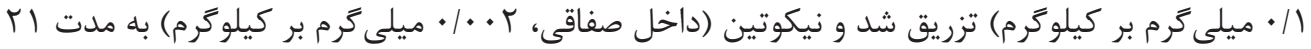

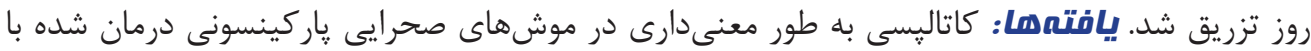

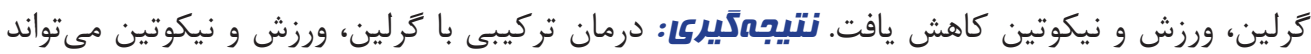

كليد وازهها:

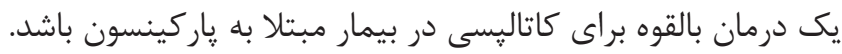
ه. بيمارى پار كينسون (1) ب. r. r.

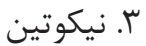

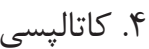

" نويسنده مسئول: رامش احمدى

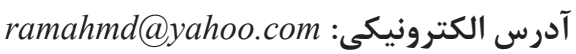


نــر مــدل پياركينسـونى توسـط رزريــن مــورد بررسـى قـرار داديسم.

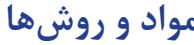

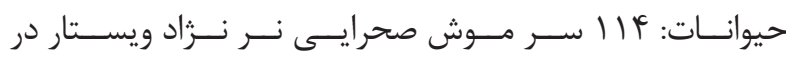

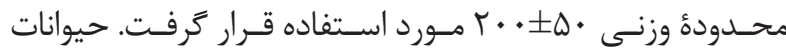

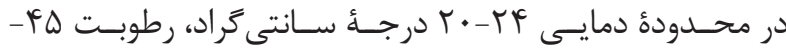

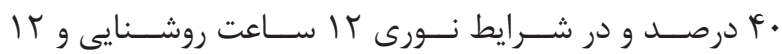

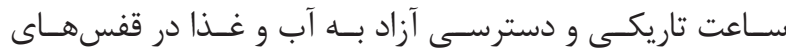

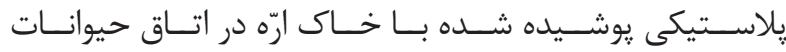

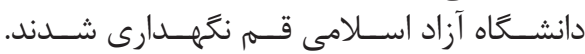

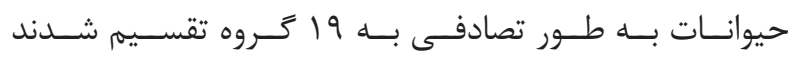

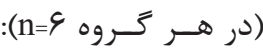

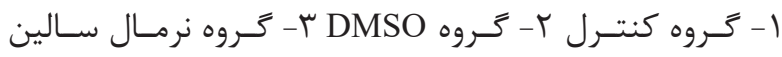

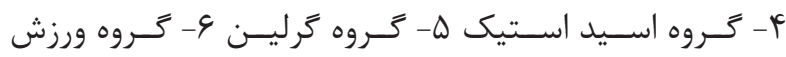

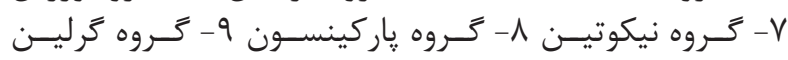

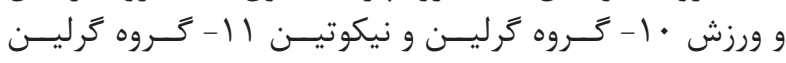

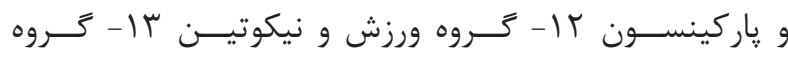

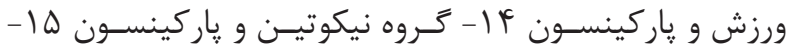

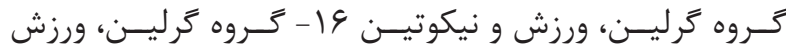

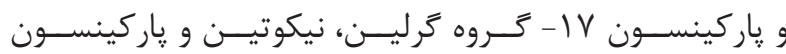

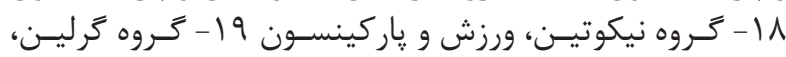

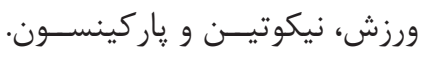

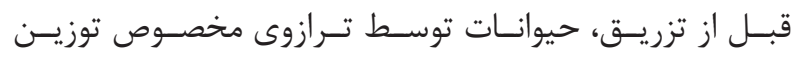

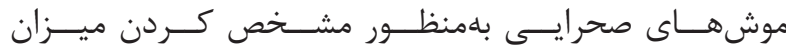

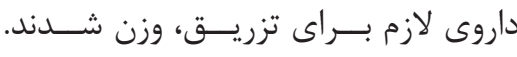

$$
\text { روش آمادهسازى داروى ترلين }
$$

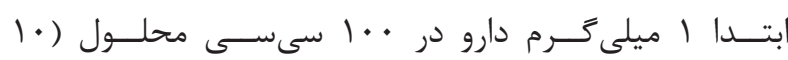

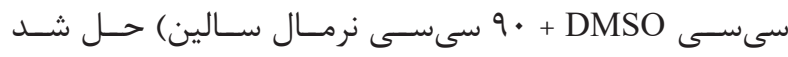

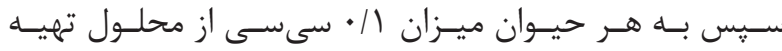

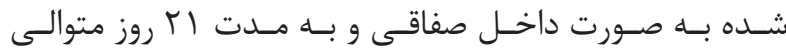

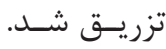

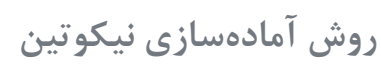

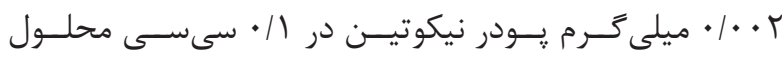

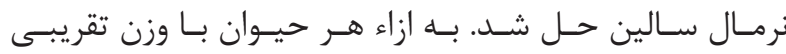

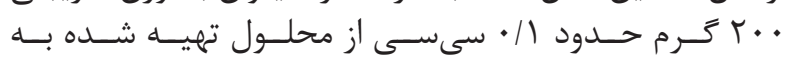

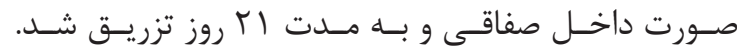

$$
\text { القاى پاركينسون }
$$

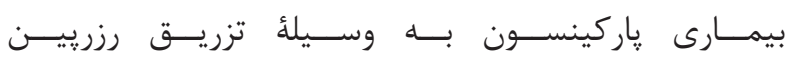
(sigma-R•AVA)

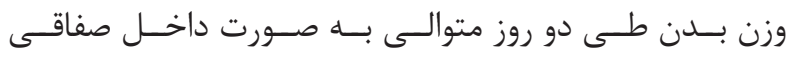

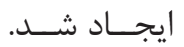

\footnotetext{
${ }^{1}$ Neurotransmitters

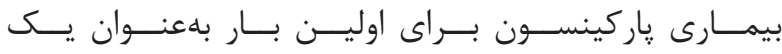

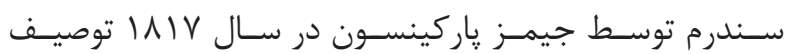

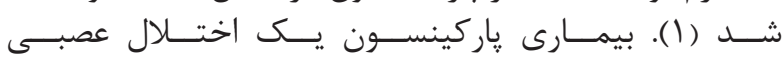

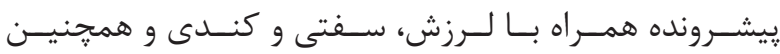

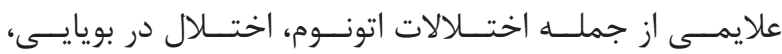

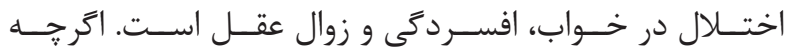

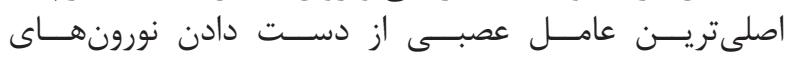

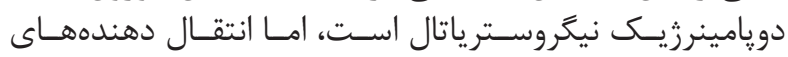

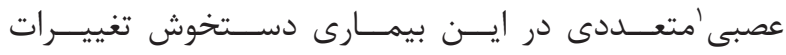

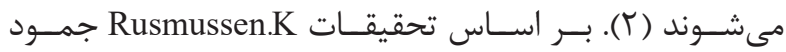

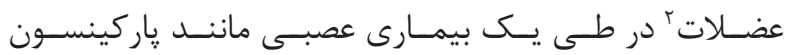

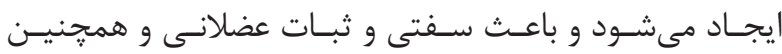

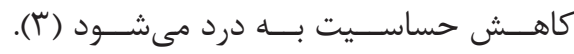

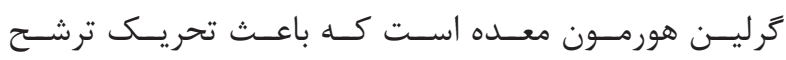

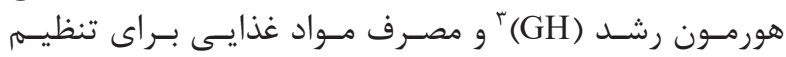

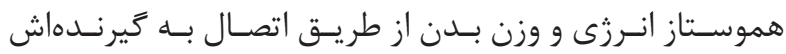

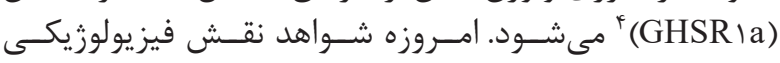

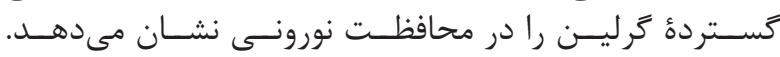

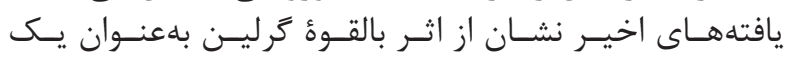

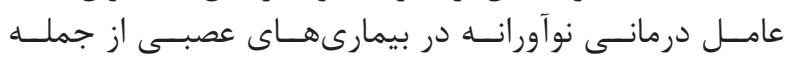

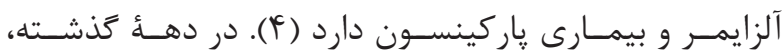

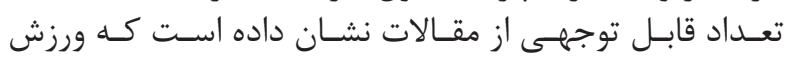

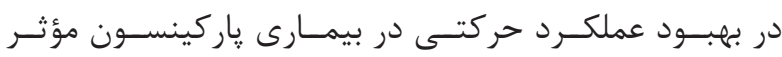

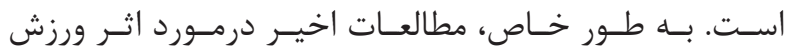

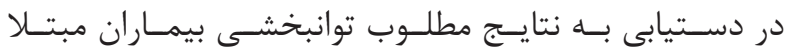

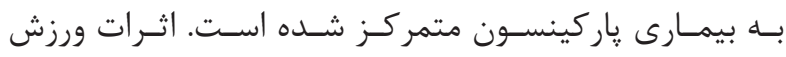

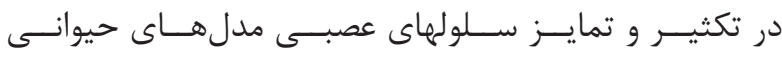

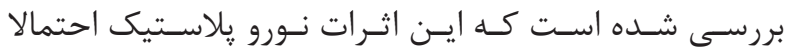

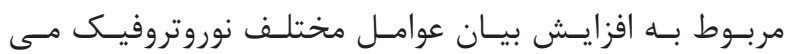

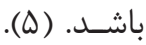

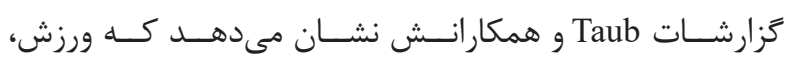

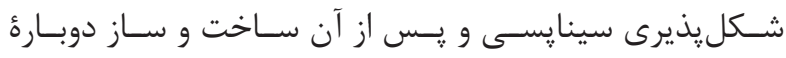

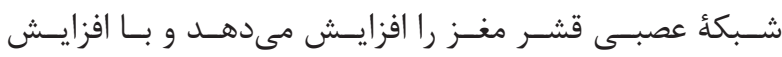

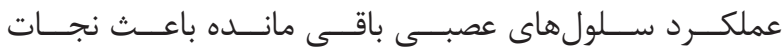

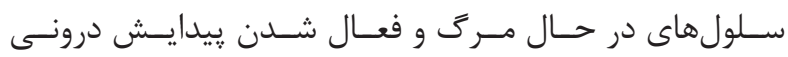

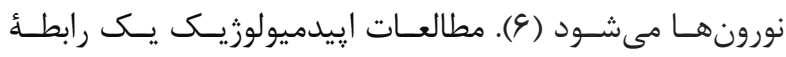

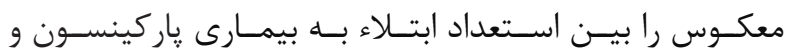

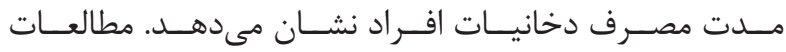

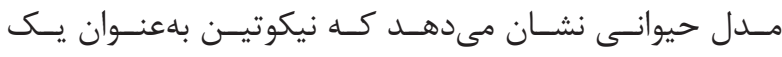

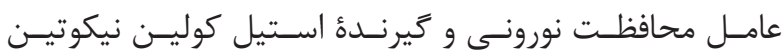

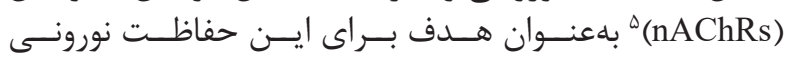

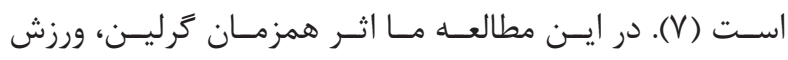

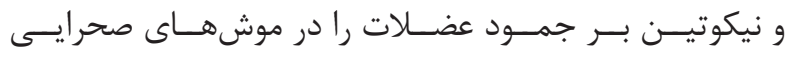

${ }^{4}$ Growth hormone secretagogue receptor

${ }^{5}$ Nicotine acetyl choline receptor 
بافتهها

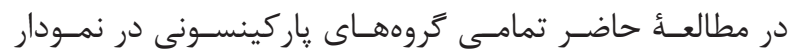

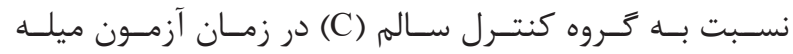

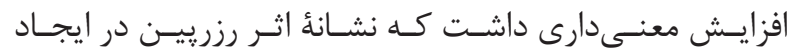

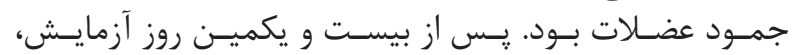

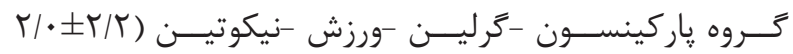

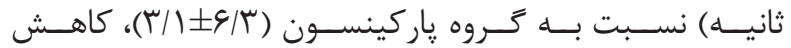

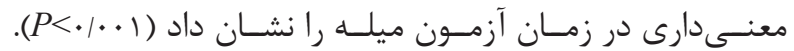

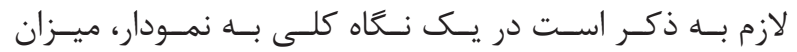

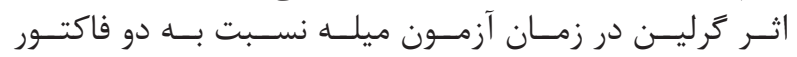

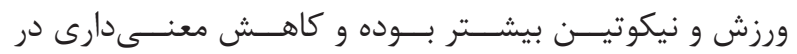

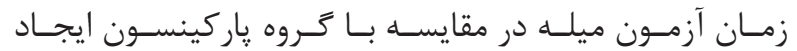

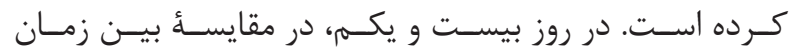

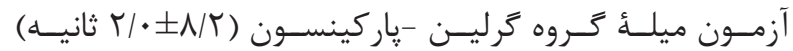

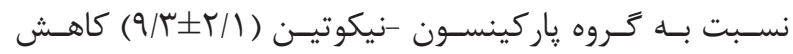

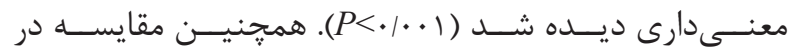

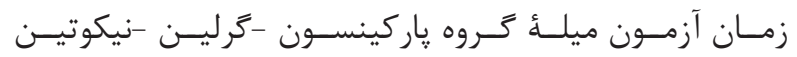

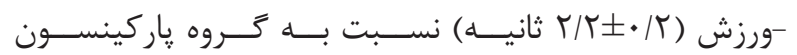

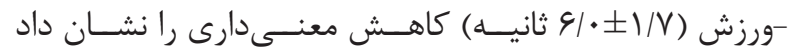
نان

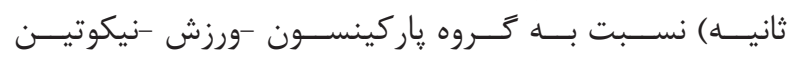

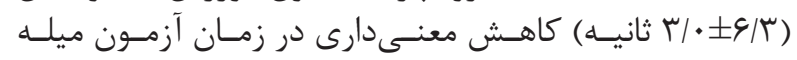

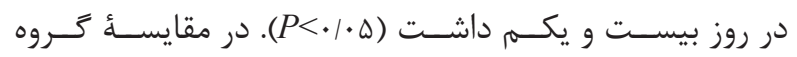

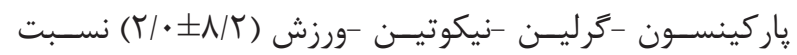

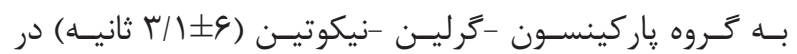

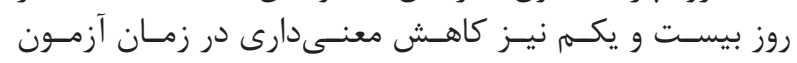

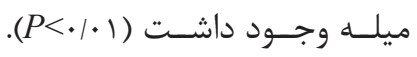

بحث و نتيجه تيرى

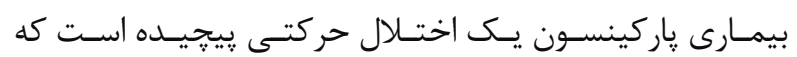

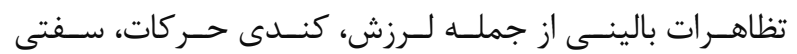

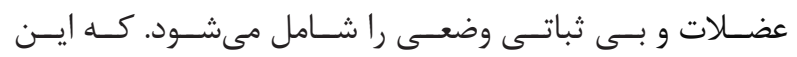

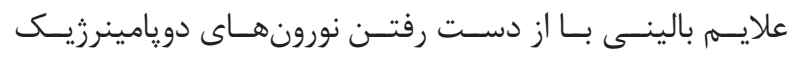

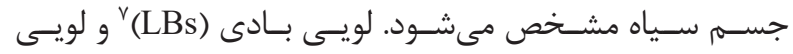

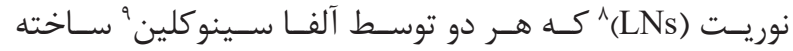

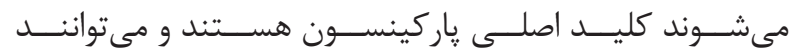

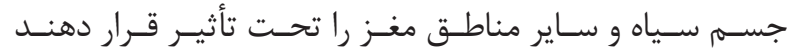

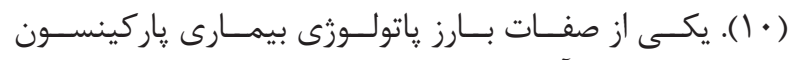

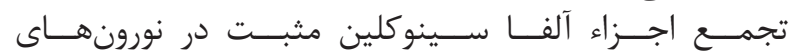

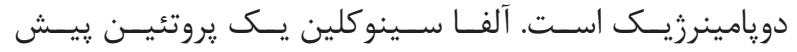

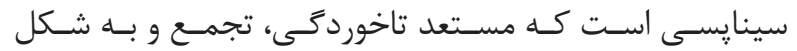

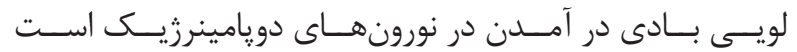

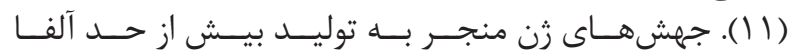

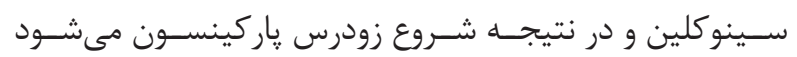

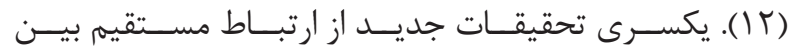

${ }^{6}$ Bar-test

${ }^{7}$ Lewy bodies
روش آمادهسازى داروى رزريين

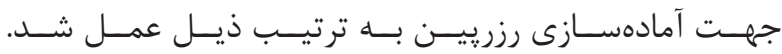

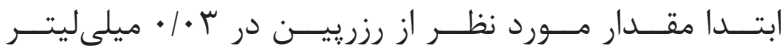

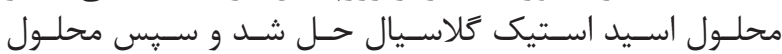

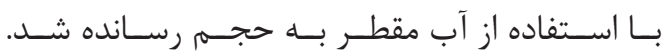
آزمون ميله

بــراى ارزيابـى جمــود عضـلات از آزمــون ميلـهـ اســتفاده شــد.

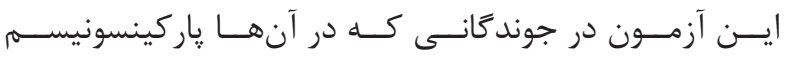

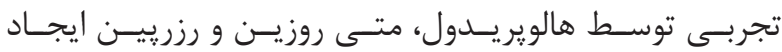

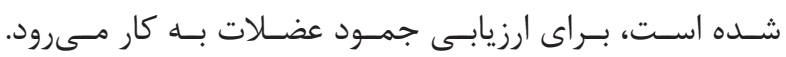

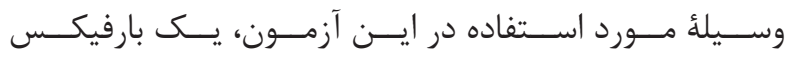

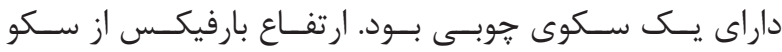

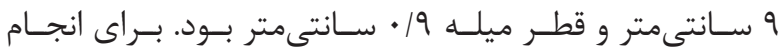

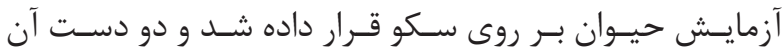

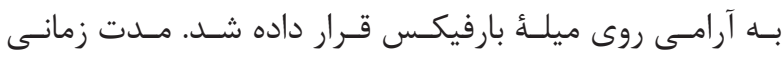

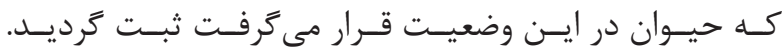

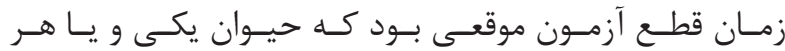

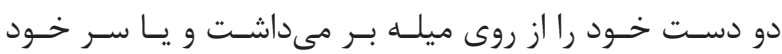

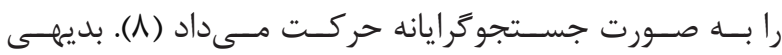

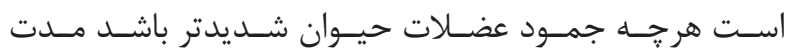

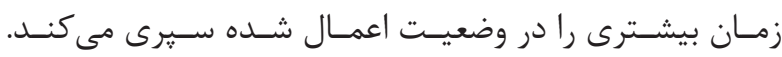

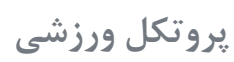

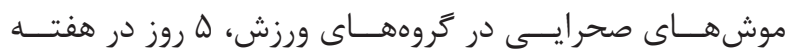

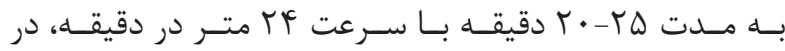

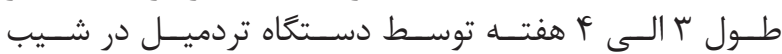

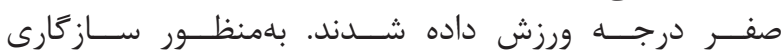

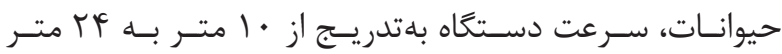

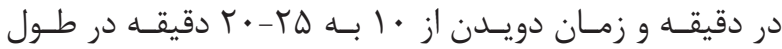

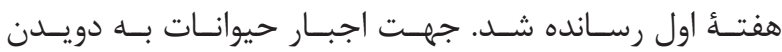

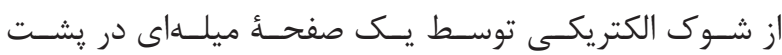

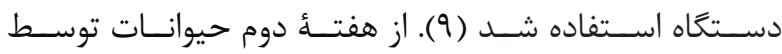

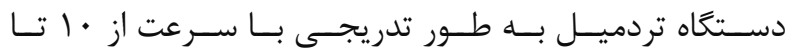

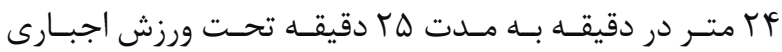
قــرار كرفتنــد. آناليز آمارى

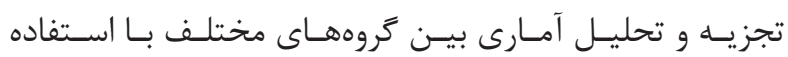

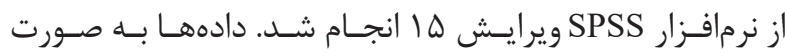

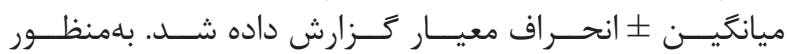

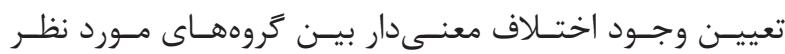

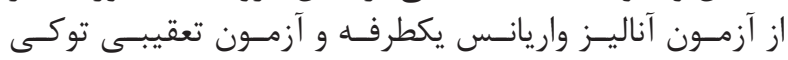

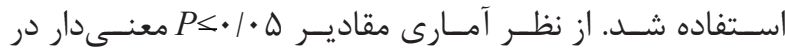

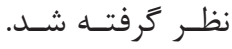

\footnotetext{
${ }^{8}$ Lewy neurites
}

${ }^{9} \alpha$-Synuclein 

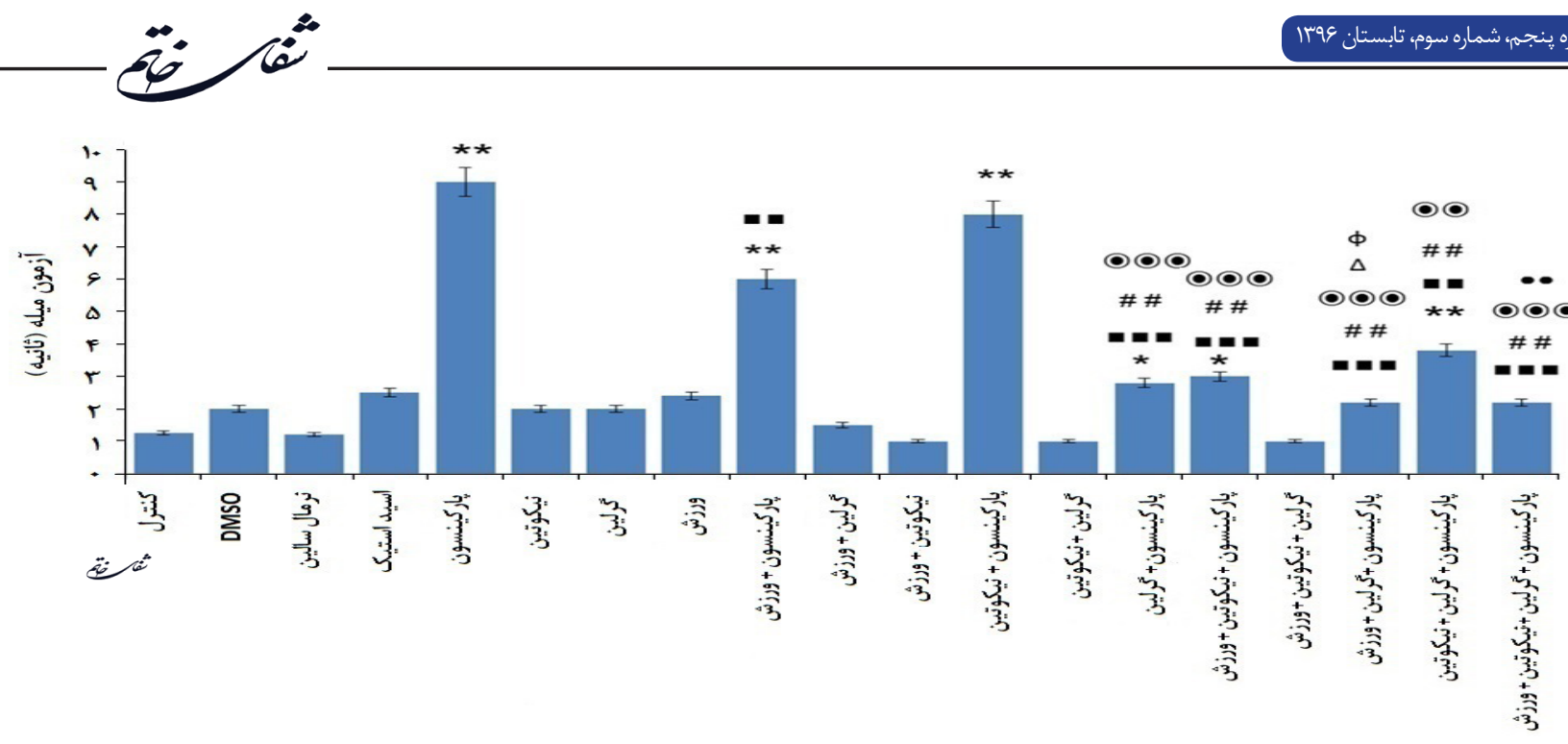

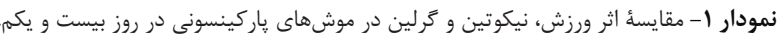

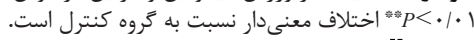

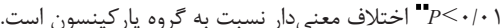

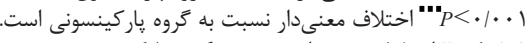

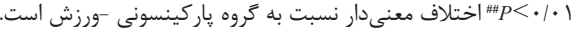

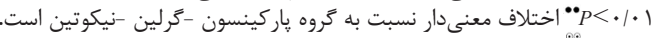

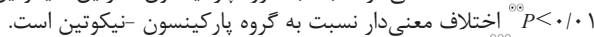

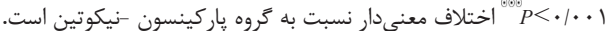

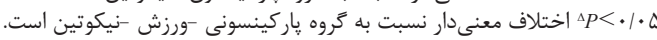

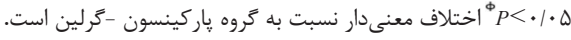

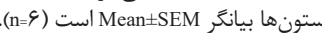

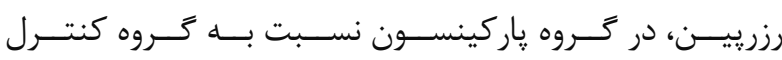

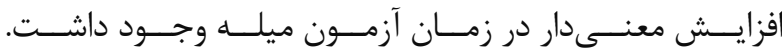

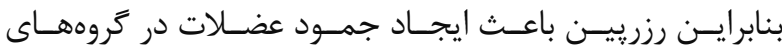

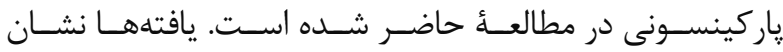

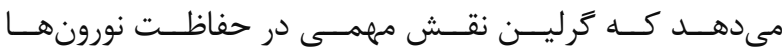

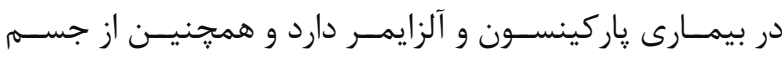

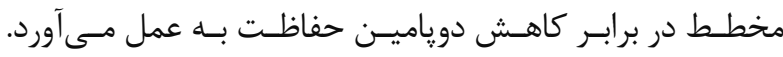

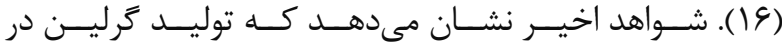

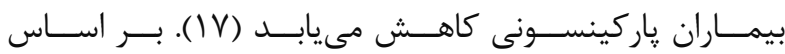

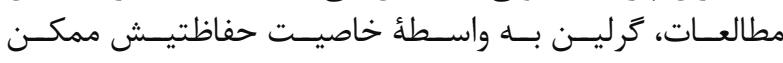

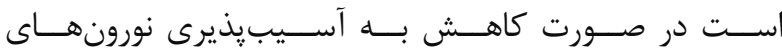

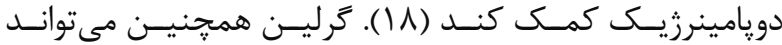

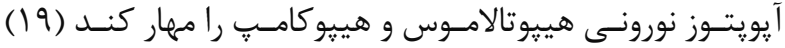

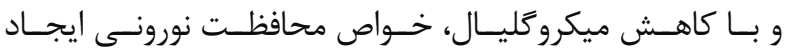

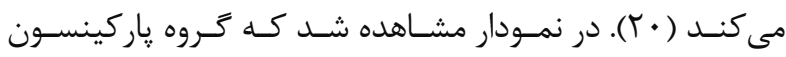

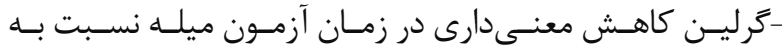

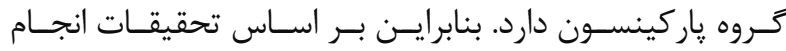

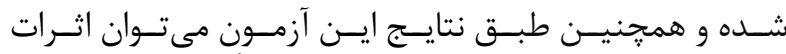

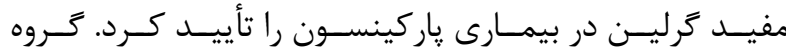

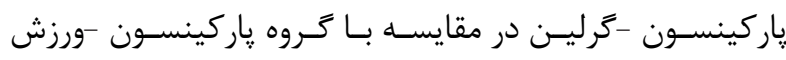

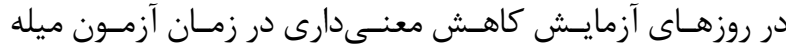

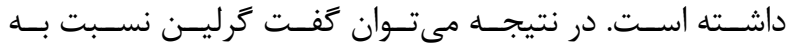

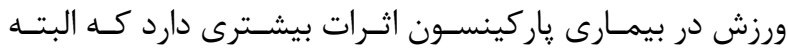

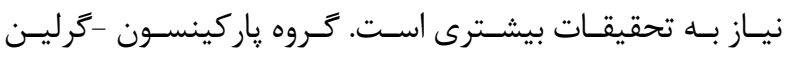

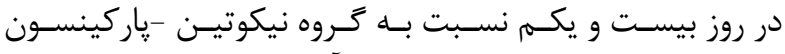

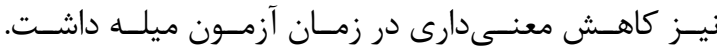

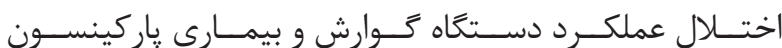

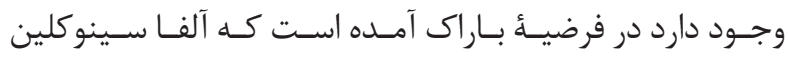

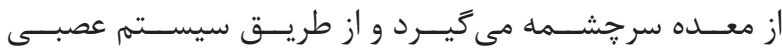

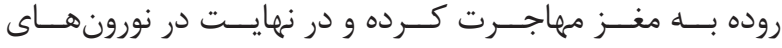

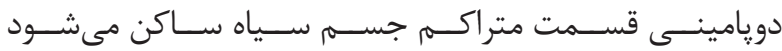

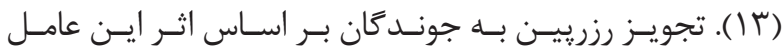

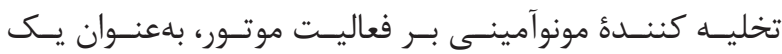

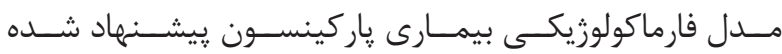

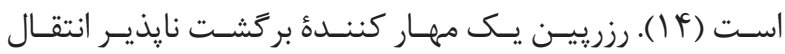

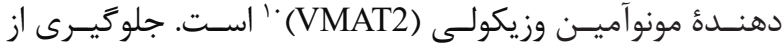

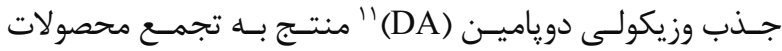

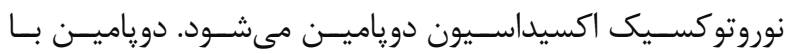

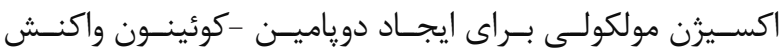

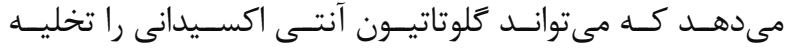

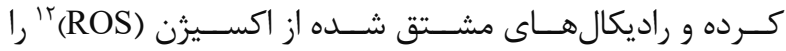

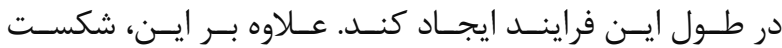

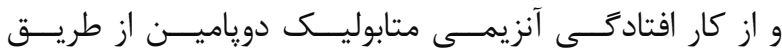

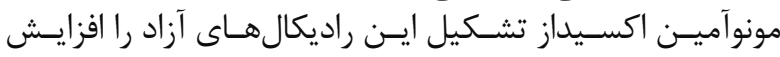

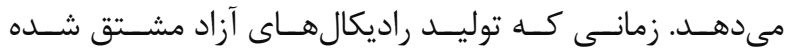

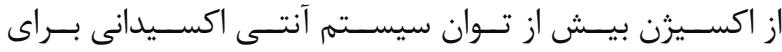

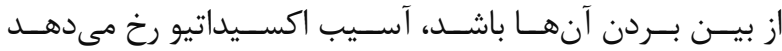

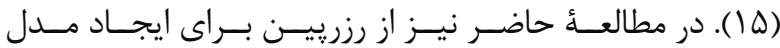

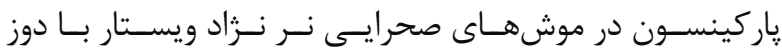

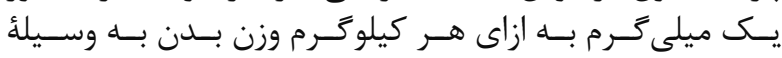

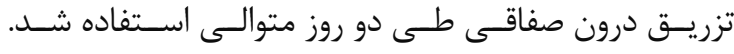

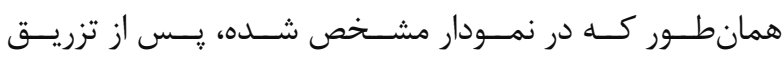

${ }^{10}$ Visicular monoamine transporter 2

${ }^{11}$ Dopamine

${ }^{12}$ Reactive oxygen speciesj 


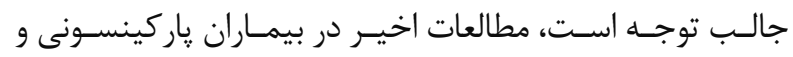

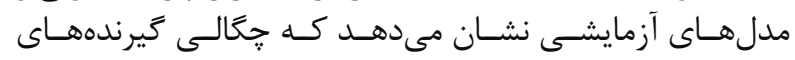

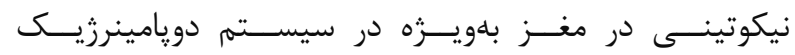

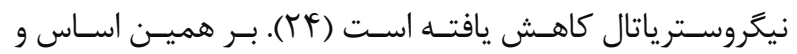

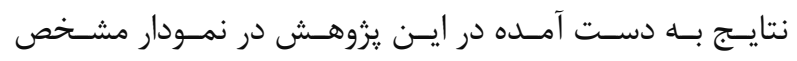

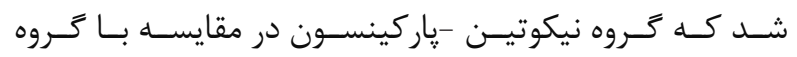

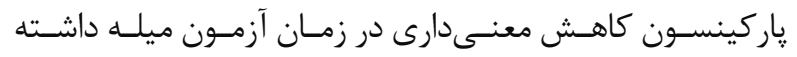

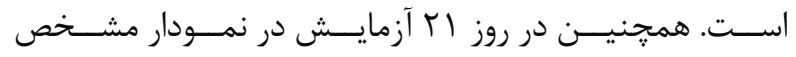

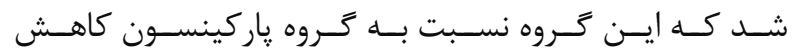

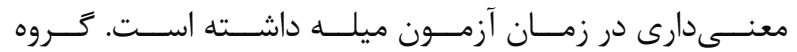

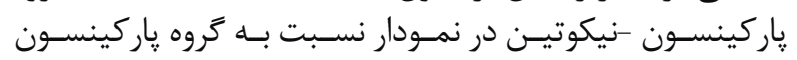

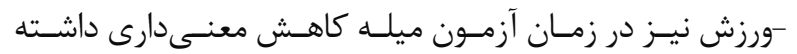

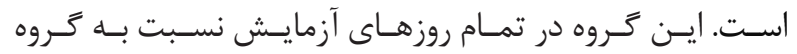

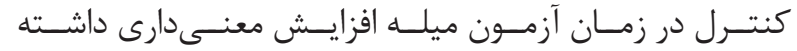

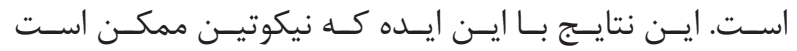

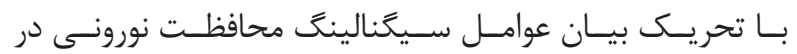

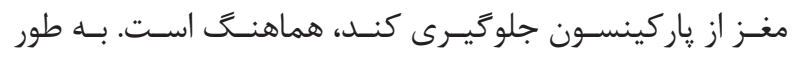

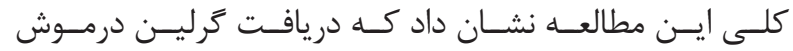

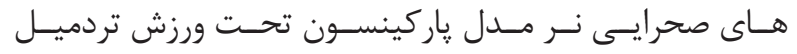

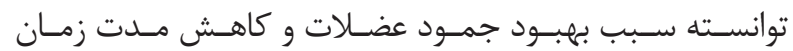

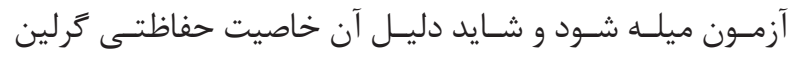

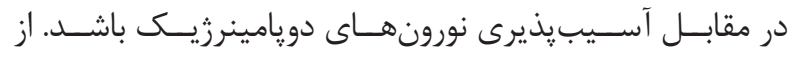

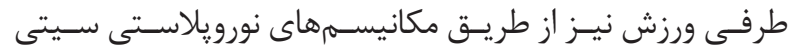

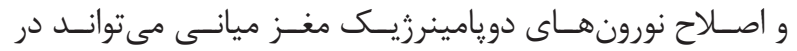

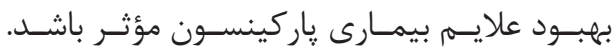

1. Sperling SA, Geneser AC, Manning CA. Parkinson's disease dementia and dementia with lewy bodies. Lichtenberg PA, Mast BT. Carpenter BD, Loebach WJ. APA handbook of clinical geropsychology. 2015. p. 227-45.

2.Quik M, Bordia T, Zhang D, Perez XA. Nicotine and nicotinic receptor drugs: potential for Parkinson's disease and drug-induced movement disorders. Int Rev Neurobiol. 2015; 124: 247-71.

3. Rasmussen K, Hsu MA, Noone S, Johnson BG, Thompson LK, Hemrick-Luecke SK. The orexin-1 antagonist SB-334876 blocks antipsychotic treatment emergent catalepsy: implication for the treatment of extrapyramidal symptoms. Schizophr Bull. 2007; 33(6): 1291-7.

4. Dos Santos VV, Rodrigues AL, De Lima TC, de Barioglio SR, Raisman-Vozari R, Prediger RD. Ghrelin as a neuroprotective and palliative agent in Alzheimer's and Parkinson's disease. Curr Pharm Des. 2013; 19(38): 6773-90.

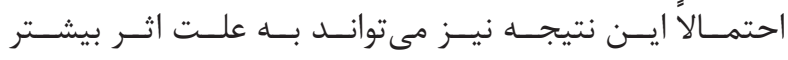

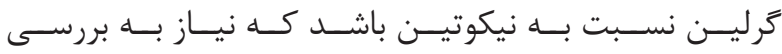

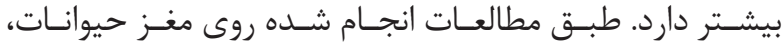

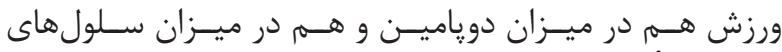

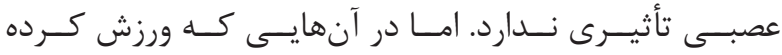

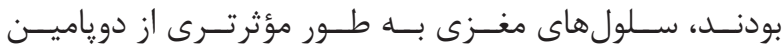

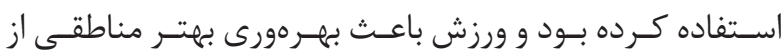

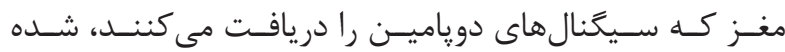

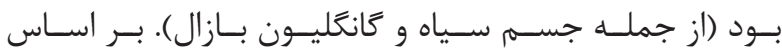

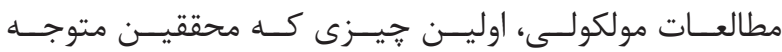

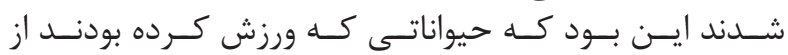

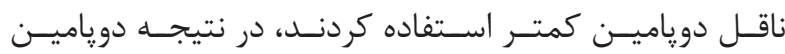

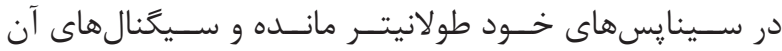

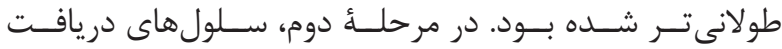

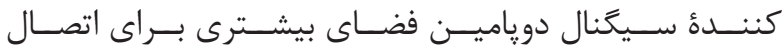

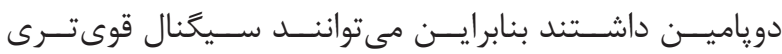

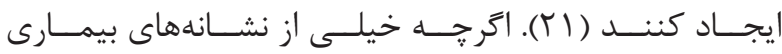

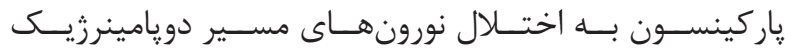

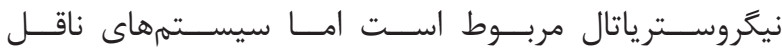

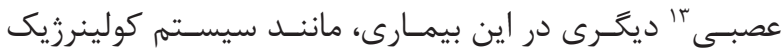

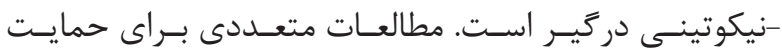

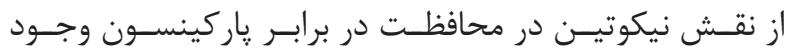

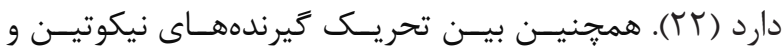

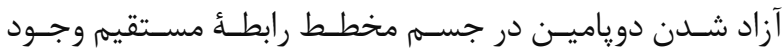

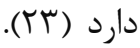

\section{منابع}

5. Frazzitta G, Balbi P, Maestri R, Bertotti G, Boveri N, Pezzoli G. The beneficial role of intensive exercise on Parkinson disease progression. Am J Phys Med Rehabil. 2013; 92(6): 523-32.

6. Taub E, Uswatte G, Elbert T. New treatments in neurorehabilitation founded on basic research. Nat Rev Neurosci. 2002; 3(3): 228-36.

7. Srinivasan R, Henley BM, Henderson BJ, Indersmitten T, Cohen BN, Kim CH, et al. Smoking-relevant nicotine concentration attenuates the unfolded protein response in dopaminergic neurons. J Neurosci. 2016; 36(1): 65-79.

8. Toy WA, Petzinger GM, Leyshon BJ, Akopian GK, Walsh JP, Hoffman MV, et al. Treadmill exercise reverses dendritic spine loss in direct and indirect striatal medium spiny neurons in the 1-methyl-4phenyl-1, 2, 3, 6-tetrahydropyridine (MPTP) mouse model of Parkinson's disease. Neurobiol Dis. 2014; 63: 201-9.

9. Singh A, Naidu PS, Kulkarni SK. FK506 as effective

\footnotetext{
${ }^{13}$ Neurotransmitter systems
} 


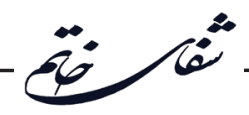

adjunct to L-dopa in reserpine-induced catalepsy in rats. Indian J Exp Biol. 2003; 41(11): 1264-8.

10. Spatola M, Wider C. Genetics of Parkinson's disease: the yield. Parkinsonism Relat Disord. 2014; 20: 35-8.

11. Uversky VN. Neuropathology, biochemistry, and biophysics of alpha-synuclein aggregation. J Neurochem. 2007; 103(1): 17-37.

12. Xilouri M, Brekk oR, Stefanis L. Autophagy and alpha synuclein: relevance to Parkinson's disease and related synucleopathies. 2016; 31(2): 178-92.

13. Braak H, de Vos RA, Bohl J, Del Tredici K. Gastric alpha-synuclein immunoreactive inclusions in Meissner's and Auerbach's plexuses in cases staged for Parkinson's disease-related brain pathology. Neurosci Lett. 2006; 396(1): 67-72.

14. de Freitas CM, Busanello A, Schaffer LF, Ricardo Peroza L, Nunes Krum B, Queiroz Leal C, et al. Behavioral and neurochemical effects induced by reserpine in mice. Psychopharmacology. 2016; 233(3): 457-67.

15. Fernandes VS, Santos JR, LEãO AH, Medeiros AM, Melo TG, Izídio GS, et al. Repeated treatment with a low dose of reserpine as a progressive model of Parkinson's disease. Behav Brain Res. 2012; 231(1): 154-63.

16. Andrews ZB. The extra-hypothalamic actions of ghrelin on neuronal function. Trends Neurosci. 2010; 34(1): 31-40.

17.Unger MM, Moller JC, Mankel K, Eggert KM, Bohne K, Bodden M, et al. Postprandial ghrelin response is reduced in patients with Parkinson's disease and idiopathic REM sleep behavior disorder: a peripheral biomarker for early Parkinson's disease? J Neurol. 2011; 258(6): 982-90.

18. Bayliss JA, Lemus M, Santos VV, Deo M, Elsworth JD, Andrews ZB. Acylated but not des-acyl ghrelin is neuroprotective in an MPTP mouse model of Parkinson's disease. J Neurochem. 2016; 137(3): 460-71.

19. Jiang H, Li LJ, Wang J, Xie JX. Ghrelin antagonizes MPTP-induced neurotoxicity to the dopaminergic neurons in mouse substantia nigra. Exp Neurol. 2008; 212(2): 532-7.

20. Moon M, Choi JG, Nam DW, Hong HS, Choi YJ, Oh MS, et al. Ghrelin ameliorates cognitive dysfunction and neurodegeneration in intrahippocampal amyloidbeta1-42 oligomer-injected mice. J Alzheimer Dis. 2011; 23(1): 147-59.

21. Giselle M, Petzinger MD. Does exercise impact Parkinson's. Parkinson's Disease Foundation. 2009; 800: 457-6676.

22. Elbaz A, Moisan F. Parkinson's disease: is there a strong environmental contribution. Rev Neural (Paris). 2010; 166(10): 757-63.

23. Pistillo F, Clementi F, Zoli M, Gotti C. Nicotinic, glutamatergic and dopaminergic synaptic transmission and plasticity in the mesocorticolimbic system: focus on nicotine effects. Prog Neurobiol. 2015; 124: 1-27.

24. Quik M, Campos C, Bordia T, Strachan JP, Zhang $\mathrm{J}$, McIntosh JM, et al. $\alpha 4 \beta 2$ nicotinic receptors play a role in the nAChR-mediated decline in 1-dopa-induced dyskinesias in parkinsonian rats. 2013; 71: 191-203. 